\title{
Detection of Regional DNA Methylation Using DNA-Graphene Affinity Interactions ${ }^{\dagger}$
}

Received 00th January 20xx, Accepted 00th January 20xx

DOI: $10.1039 / x 0 x \times 00000 x$

www.rsc.org/

We report a new method for the detection of regional DNA methylation using base-dependent affinity interaction (i.e., adsoption) of DNA with graphene. Due to the strongest adsoption affinity of guanine bases towards graphene, bisulfite-treated guanine-enriched methylated DNA leads to a larger amount of the adsorbed DNA on the graphene-modified electrodes in comparison to the adenine-enriched unmethylated DNA. The level of the methylation is quantified by monitoring the differential pulse voltammetric current as a function of the adsorbed DNA. The assay is sensitive to ditinguish methylated and unmethylated DNA sequences at single $\mathrm{CpG}$ resolution by differentiating changes in DNA methylation as low as $\mathbf{5 \%}$. Furthermore, this method has been used to detect methylation levels in a collection of DNA samples taken from oesophageal cancer tissues.

DNA methylation is a cell-type specific epigenetic marker, essential for controlling gene expression via transcriptional regulation, silencing of repetitive DNA and genomic imprinting. ${ }^{1}$ Current advances in DNA methylation research have suggested that different types of cancers appear to have distinct DNA methylation levels at selected regions (i.e., regional methylation), which are responsible for their various responses to treatment. ${ }^{2}$ For example, regional methylation plays a fundamental role in the initiation and progression of oesophageal squamous cell carcinoma (ESCC) by inactivating transcription and loss of gene function. ${ }^{3}$ Also, DNA methylation based biomarker has proven to be used alone or in combination with other diagnostic methods in cancer. ${ }^{3 \mathrm{~d}}$ Thus, detection of methylation in targeted cancer specific genes could have diagnostic and prognostic implications in human cancers.

\footnotetext{
Cancer Molecular Pathology Laboratory in School of Medicine, Menzies Health Institute Queensland, Griffith University, Gold Coast Campus, Australia

${ }^{b}$. School of Natural Sciences, Griffith University, Nathan Campus, QLD 4111,

Australia

c. The University of Queensland, Brisbane, QLD 4072, Australia

d. Queensland Micro and Nanotechnology Centre, Griffith University, Nathan Campus, QLD 4111, Australia

Corresponding author emails: v.gopalan@griffith.edu.au(VG); a.lam@griffith.edu.au (AKL) and m.shiddiky@griffith.edu.au (MJAS).

+Electronic Supplementary Information (ESI) available: Experimental details [phage display data]. See DOI: 10.1039/x0xx00000x
}

Over the past several decades, a number of conventional molecular biology approaches, including-bisulfite sequencing and real time quantitative polymerase chain reaction (RTqPCR), have been extensively used to measure the level of DNA methylation in cancers. ${ }^{2,4}$ These approaches employed sequencing, mass spectrometric or fluorescence readouts to differentiate between methylated and unmethylated sequences. They are relatively robust but are limited by the costly instruments, fluorescent labels and long analysis time. Furthermore, these methods are limited by the background fluorescence interference as well as to high labour and bioinformatics costs.

In the recent years, much attention has been focused on developing inexpensive and faster detection strategies based on colorimetry, electrochemistry, Raman scattering readouts. ${ }^{2,5-7}$ While all these methods have many advantages, their sensor fabrication procedure and data deconvolution methods are rather complicated due to the involvement of complex surface functionalisation steps or coupling chemistry. ${ }^{2,5-7}$ Therefore, a simple and inexpensive method that could simplify the detection method by avoiding the complicated chemistry underlying each step of the sensor fabrication represents an appealing alternative to alleviate some of these issues.

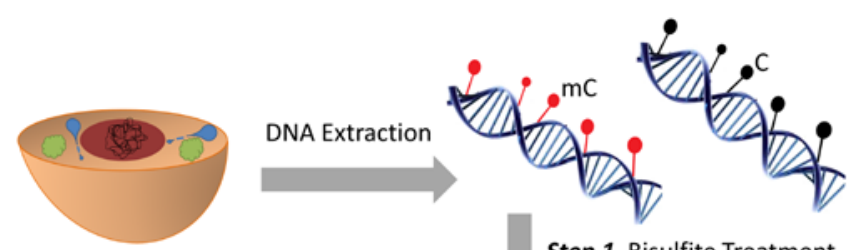

Cell from cancerous tissue Step 1 Bisulfite Treatment Step 2 Asymmetric PCR

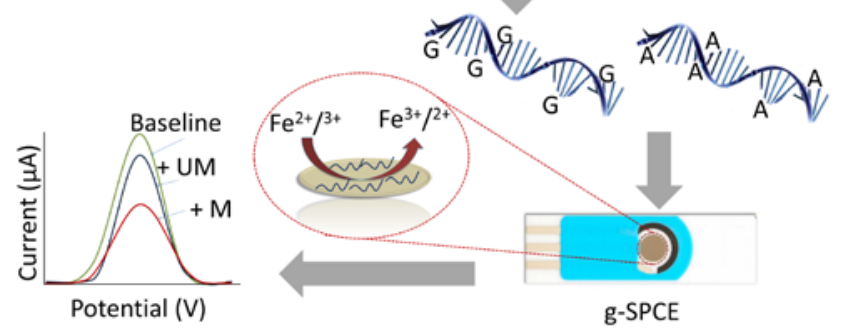

More recently, we have reported a simple method for quantifying DNA methylation using different adsorption affinity of DNA bases onto an unmodified gold substrate. ${ }^{8}$ Similar to gold substrate, graphene $e^{9}$ and graphene oxide ${ }^{10}$ have been reported as promising substrates for adsorbing nucleobases and nucleosides. Over the past few years, several fundamental studies have been carried out to explore the nature of the direct interaction (i.e., adsorption) of nucleobases and nucleosides onto the graphene and graphene oxide surface. ${ }^{9-11}$ These studies have suggested that the physisorption between individual nucleobases and the graphene (i.e., adsorption on graphene) is controlled by the polarisabilities of the individual nucleobases. Among all nucleobases, guanine and adenine with their five- and sixmembered rings possess the largest polarisabilities, whereas other bases with only six-membered rings exhibit lower 
polarisabilities. Additionally, guanines with its double-bonded oxygen atom possess a larger polarisability than adenine. Since the van der Wall (vdW) energy is directly proportional to the interacting nucleobases, Scheicher ${ }^{11}$ and Rao $^{9}$ have proposed that vdW interaction is indeed the main driving force for the adsorption of nucleobases onto the graphene and follows the adsorption trend as guanine $(G)>$ adenine $(A)>$ thymine $(T)>$ cytosine (C). Because this interaction is base (i.e., sequence) dependent and bisulfite conversion generates two DNA sequences with different base compositions, bisulfiteconverted two DNA sequences should give different adsorption patterns on graphene surface. To date, there is no other method that uses the graphene-DNA affinity interaction to quantify DNA methylation.

Here we report a simple and inexpensive method for detecting regional DNA methylation using direct adsorption of bisulfite-treated and PCR amplified DNA sequences onto graphene-modified screen-printed carbon electrode (g-SPCE). Fig. 1 illustrates the principle of the method. Briefly, ds-DNAs were first extracted from the cancer cell lines (see ESIt for details). A bisulfite conversion step was then performed to convert unmethylated cytosines in ds-DNA (double stranded DNA) into uracils while methylated cytosines remain unchanged. In a subsequent asymmetric PCR amplification step, all ds-DNA were converted to ss-DNA (single stranded DNA) amplicons. In this step, cytosines in the complementary strand will be copied to guanines and uracils to adenines resulting in methylated sample into guanine-enriched and unmethylated into adenine-enriched ss-DNA. These samples were then directly adsorbed on the g-SPCE surface. The adsorbed ss-DNA amplicons were detected by differential pulse voltammetry (DPV) in the presence of a small redox active $\left[\mathrm{Fe}(\mathrm{CN})_{6}\right]^{3-/ 4-}$ group. Previously, we ${ }^{8}$ and Zhang et al. ${ }^{12}$ have shown that unlike conventional redox system (e.g., $\left.\left[\mathrm{Ru}\left(\mathrm{NH}_{3}\right)_{6}\right]^{3+} /\left[\mathrm{Fe}(\mathrm{CN})_{6}\right]^{3-}\right)^{13}$, the $\left[\mathrm{Fe}(\mathrm{CN})_{6}\right]^{3-/ 4-}$ alone is sufficient for quantifying surface-bound sequences reliably. As outlined by Zhang et al. ${ }^{12}$, the process follows an electron transfer kinetics-based mechanism, where density of the adsorbed DNA sequences at the electrode surface should be sufficiently low (i.e., partial blocking). Under this condition, the columbic repulsion between the surface-bound ss-DNA and $\left[\mathrm{Fe}(\mathrm{CN})_{6}\right]^{3-}$ is not strong enough to fully repel $\left[\mathrm{Fe}(\mathrm{CN})_{6}\right]^{3-}$ molecules from accessing the surface. Therefore, it generates a significant DPV signal which is lower than that of the un-modified electrode (i.e., before ss-DNA adsorption). ${ }^{8}$ In the current method, since the adsorption strength of DNA bases towards graphene vary as $\mathrm{G}>\mathrm{A}>\mathrm{T}>\mathrm{C}$, the guanine-enriched unmethylated DNA leads to a larger level of adsorbed DNA on the electrodes in comparison to the adenine-enriched methylated DNA. Hence, as schematically shown in Fig.1, unmethylated DNA results in a smaller DPV current (i.e., larger relative current change, $\% I_{\text {Relative; }}$ see ESIt for details).

We have first explored the oncogenic properties and altered expression of $F A M 134 B$ (JK1) gene in oesophageal squamous cell carcinoma. ${ }^{14 a}$ Also, FAM134B has been reported to have roles in pathogenesis as well as prediction of prognosis of other gastrointestinal cancer, namely colorectal cancer. ${ }^{14 \mathrm{~b}}$ Most

Fig. 1 Schematic of graphene-DNA adsorption based methylation assay. The adsorption of ss-DNA on g-SPCE repulse $\left[\mathrm{Fe}(\mathrm{CN})_{6}\right]^{3-}$ molecules from accessing electrode surface, providing a significate DPV signal. Inset, typical differential pulse voltammetric signals showing the guanine-enriched methylated DNA that produces lower DPV currents in comparison to the adenine-enriched unmethylated DNA.

current approaches detect DNA methylation in oesophageal cancers via methylation specific PCR amplification process. ${ }^{15}$ In this proof-of-concept study, we have used graphene-DNA affinity interaction for detecting gene-specific DNA methylation in FAM134B. Firstly, the FAM134B promoter region containing $11 \mathrm{CpG}$ sites located within a length of 60 bases was chosen as a target DNA. In order to further validate our approach, we have designed synthetic samples containing $0,1,5$ and $11 \mathrm{CpG}$ sites within the promoter region of FAM134B gene which mimic the bisulfite treated and asymmetric PCR processes methylated and methylated DNA regions (See Table S1, ESI†).

One of the key considerations of the current method is that there is only a finite amount of electrode area and we should have the right amount of adsorbed DNA on the electrode surface so that the extent of methylation makes discernible signal difference. Therefore, we first optimised adsorption time and $\mathrm{pH}$ of the solution to achieve optimal amount of adsorbed DNA on the electrode surface so that the affinities of the DNA with different amounts of methylation can be discerned. Relative current differences $\left(\Delta I_{\text {Relative, }}\right.$ see see $\left.E S I+\right)$ between the $100 \mathrm{nM}$ methylated ( $11 \mathrm{CpG}$ ) and unmethylated (0 CpG) DNA samples were measured. As seen in Fig. S1, an adsorption time of only $1 \mathrm{~min}$ is sufficient to generate a significant $\Delta I_{\text {Relative }}$ value. The maximum $\Delta /_{\text {Relative }}$ was found at 2 min, which was rapidly decreased with increasing adsorption time. Long adsorption time (>10 $\mathrm{min}$ ) would lead to large amount of adsorbed DNA (i.e., complete block of the surface) on electrode surface. , This results in a similar level of columbic repulsion between the surface-bound DNA and $\left[\mathrm{Fe}(\mathrm{CN})_{6}\right]^{3-}$ for both the methylated and unmethylated cases, providing two DPV signals with almost identical magnitudes leading to a small $\Delta I_{\text {Relative }}$ changes.

We then evaluated the $\mathrm{pH}$-dependent $\Delta I_{\text {Relative }}$ changes by varying the $\mathrm{pH}$ of the solution from 3.0 to 9.5 (Fig. S2). Clearly, both methylated and unmethylated DNA samples could be distinguished at all the $\mathrm{pH}$ examined. The optimal $\Delta /_{\text {Relative }}$ value

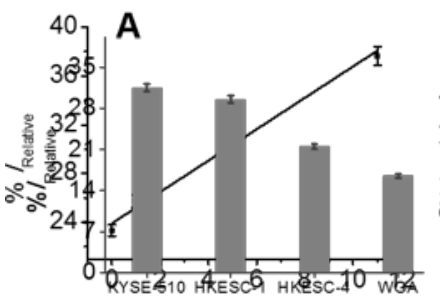

Number of reethylated CpG sites

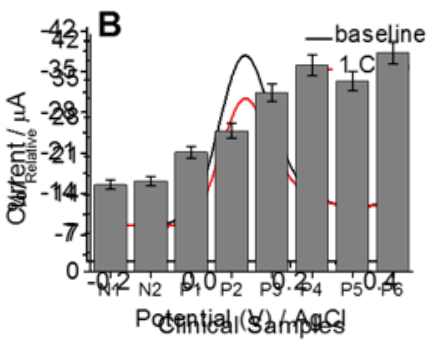

Fig. 2 Left, Differential pulse voltammetric current changes with respect to the designated $\mathrm{CpG}$ sites. Right, typical DPV signal for the sample containing $1 \mathrm{CpG}$ site and its corresponding background signal. Each data point represents the average of three repeat trails, and error bars represent the standard deviation of measurements (\%RSD $=<5 \%$ for $n=3$ ). 
was found at $\mathrm{pH}$ 7.4. This can be explained by the fact that negative charge of the phosphate backbone of DNA at this $\mathrm{pH}$ is optimal to hinder the graphene-DNA interaction for both methylated and unmethylated samples while still allowing the methylated DNA with higher guanine contents to be interacted strongly. At the lower $\mathrm{pH}$, the N3 position of the cytosines in methylated and unmethylated sequences can be protonated $(\mathrm{pKa}=4.2) .^{10}$ This may contribute to reduce the Van-der Wall interaction (i.e., electrostatic repulsion) between targets sequences and graphene surface, and thus result in a lower adsorption, leading to higher DPV currents (i.e., lower $\Delta I_{\text {Relative }}$ changes). At the higher $\mathrm{pH}$, the electrostatic interaction (adsorption) between graphene and DNA sequences is very strong, which significantly contribute to facilitate their adsorption on graphene-modified surfaces (i.e., both targets reach to the saturation level within a very short time giving a reduced $\Delta I_{\text {Relative }}$ value).

The main advantage of gold standard sequencing-bisulfite based method is that they can detect DNA methylation with single-base resolution. To evaluate the feasibility of our assay for detecting a minimum number of $\mathrm{CpG}$ methylation, four synthetic DNA samples containing $0,1,5$ and $11 \mathrm{CpG}$ sites were

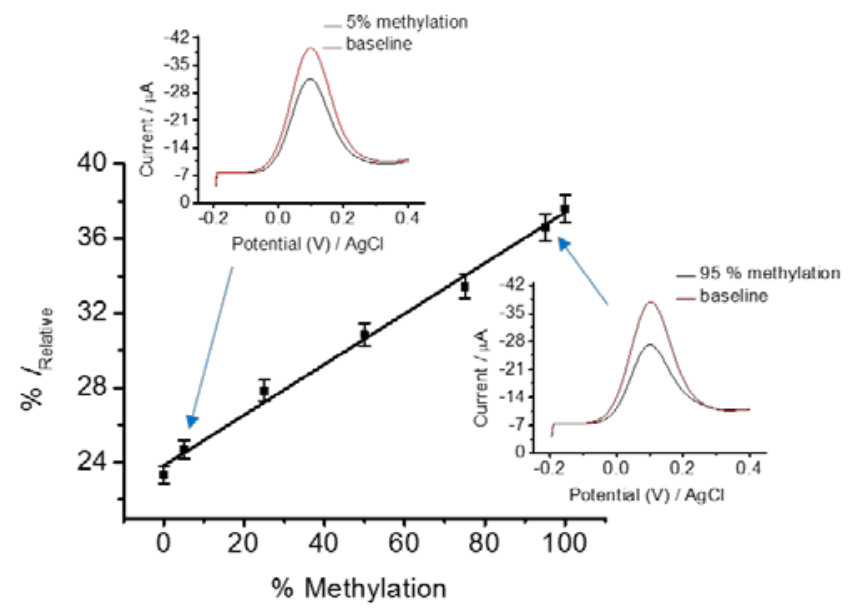

Fig. 3 Differential pulse voltammetric current changes with respect to the designated methylation level. Inset, typical DPV signals for the sample containing $5 \%$ (left, top) and 95\% (right, bottom) and their corresponding background signals. Each data point represents the average of three repeat trails, and error bars represent the standard deviation of measurements ( $\%$ RSD $=<5 \%$ for $n=3$ ).

Fig. 4 Relative current changes for detecting $F A M 134 B$ promoter region in (A) three oesophageal cancer cell lines and unmethylated, and (B) two normal (N1 and N2) and six (P1-P6) oesophageal cancer tissue samples. Each data point represents the average of three repeat trails, and error bars represent the standard deviation of measurements (\%RSD $=<5 \%$ for $n=3$ ).

tested (as outlined above and in Table S1, ESIT). Fig. 2 shows that the decrease of the relative current changes is a function of the number of CpG cites. This is due to the increase of the guanine contents with increasing methylated $\mathrm{CpG}$ sites in the target sequence. The level of relative absorption pattern of methylated CpG sites agreeably implies the high specificity of our assay for effective detection of DNA methylation at a single CpG level of resolution.
Since tissue samples from patients with cancer usually contain a mixture of methylated and unmethylated DNA, accurate quantification of heterogeneous DNA methylation pattern is significant for detection and prediction of various clinicopathological parameters in cancers. It is therefore important to detect the degree of methylation in a high background of unmethylated DNA samples. To evaluate the assay performance for detecting heterogeneous DNA methylation pattern, we analysed the dependence of the relative current changes on various degree of methylation. The samples were prepared by mixing synthetic standards of methylated and unmethylated DNA sequences to get $0 \%, 5 \%$, $25 \%, 50 \%, 75 \%, 95 \%$ and $100 \%$ methylation, Fig. 3 . The relative current changes $\left(\% I_{\text {Relative }}\right)$ increases with increasing levels of methylation, probably due to the increasing guanine contents which results in lowering the DPV current and thus the higher

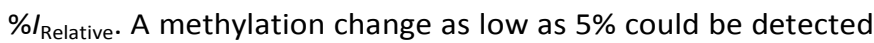
from $100 \mathrm{nM}$ of DNA. These data was much better than our previous gold-DNA based approach ${ }^{8}$ and was of comparable to recent approaches, ${ }^{5,6}$ and clearly demonstrate that our approach is both specific and sensitive in detecting methylated DNA in the nanogram regime.

To test the applicability of our assay for detecting methylation level of $F A M 134 B$ promoter gene, DNA samples derived from three ESSC cell lines were tested, Fig. 4A. A fully unmethylated whole genome amplified DNA was used as an internal standard. For avoiding any PCR bias, as outlined in our previously reported methylasorb assay, ${ }^{8}$ we normalized the gene copy number prior to PCR amplification (see ESIt for details). As expected, for all the three cancer cell lines and WGA samples, significant relative current changes were observed indicating the presence of different degree of methylation. Importantly, two of the cell-derived samples (i.e., KYSE-510 and HKESC-1) resulted in a large relative current changes when compared to that of the unmethylated WGA (e.g., $\Delta I_{\text {Relative }}$ between KYSE-510 cell lines and unmethylated WGA was found to be $\sim 14.6 \%$ ), indicating DNA sequence derived from KYSE-510 and HKESC-1 cell lines could be hypermethylated at $F A M 134 B$ promoter gene. These data clearly indicate that the proposed assay may be a useful alternative for detecting $F A M 134 B$ promoter gene methylation in cell-derived samples.

To further demonstrate the applicability of our method in analysing clinical samples, we extended our assay to analyse six tissue DNA samples derived from patients with primary oesophageal squamous cell carcinoma. Two oesophageal noncancerous tissue DNA samples were also used as a control (see ESIt for details). Fig. 4B indicates that all samples showed different degree of methylation. The level of relative current changes with respect to that of the WGA obtained for two normal samples clearly show that these two samples are unmethylated. Also by comparing the level of relative current changes found in cell lines (Fig. 4A), we can estimate that four DNA samples derived from P3, P4, P5 and P6 cancer patients were hypermethylated, while $\mathrm{P} 1$ and $\mathrm{P} 2$ samples were hypomethylated at $F A M 134 B$ promoter gene.

Previously, it has been reported that gene silencing due to promoter hypermethylation play a fundamental role in 
pathogenesis of oesophageal squamsou cell carcinoma. ${ }^{3 a}$ To evaluate whether the promotor hypermethylation of the $F A M 134 B$ gene is linked with oesophageal cancer development, the $F A M 134 B$ mRNA expression in all studied samples were examined via GRT-PCR (see Fig. S3 and experimental details in $\mathrm{ESI}+$ ). While our assay have showed aberrant promoter hypermethylation of the FAM134B gene in four primary cancer patients ( $P 3, P 4, P 5$ and $P 6)$, the mRNA expression study revealed under-expression in three (P4, P5 and P6) of these DNA samples. Furthermore, mRNA over expression was found in three patient samples (P1, P2, P3), indicating that the associated $\mathrm{CpG}$ region could be unmethylated. In our assay, we have noted that the levels for P1 and P2 were in agreement with these data. In contrast, P3 showed significantly higher methylation levels than healthy and WGA samples. This outcome suggests that this region could be hypermethylated, in disagreement with our mRNA expression data. These data establish a correlation between promoter methylation level and mRNA expression of FAM134B gene in oesophageal squamous cell carcinoma, and validate the accuracy of our assay. Although an in-depth study is needed to fully evaluate and validate clinical utility of the method, the analytical performance of the assay in its current form suggests the high feasibility of our assay in clinical sample analysis.

In conclusion, we have developed a simple and new method for the quantification of gene-associated DNA methylation using affinity interaction between DNA bases and graphene. The method is based on the different absorption affinity of DNA nucleotides towards graphene-modified electrodes. The detection was achieved by the direct adsorption of two sequentially different DNA samples (bisulfite-treated and PCR amplified sequences representing methylated and unmethylated DNA) onto a graphene-modified electrode, which avoids multiple modifications and functionalisation steps involved in conventional assays. Furthermore, it avoids the need for sequencing analysis. Most importantly, we have tested the feasibility of our assay to detect methylation target of FAM134B promoter gene in a panel of ESCC cell lines and clinical samples from ESCC patients. We anticipated that our assay might be able to detect global hypomethylation since the methylated and unmethylated DNA-base changes of bisulfite treated genomes entails a large number of CpG sites, which might generate a marked absorption difference between fully methylated and partially methylated samples. In addition, our assay could be viably useful in clinical diagnostics because of its potential for accurate detection of epigenetic biomarker.

This work was supported by the NHMRC CDF (APP1088966 to M.J.A.S.), Griffith University New Researcher Grant Scheme and higher degree research scholarships (GUIPRS and GUPRS scholarships to M.H.H. and M.N.I.) from the Griffith University.

\section{Notes and references}

1. (a) P. A. Jones, D. Takai, Science 2001, 293, 1068-1070. (b) S. C. Wu, Y. Zhang, Nat. Rev. Mol. Cell. Biol. 2010, 11, 607620.
2. (a) Z. Taleat, K. Mathwig, E. J. R. Sudhölter, L. Rassaei, TracTrends Anal. Chem.2015, 66, 80-89. (b) L. Zhang, Y. -Z. Xu, et al. Trac-Trends Anal. Chem. 2015, 72, 114-122.

3. (a) M. L. Wong, Q. Tao Q, et al., Int. J. Oncol.2006, 28, 767773. (b) H. W. Chang, et al., Cancer 2002, 94, 386-392. (c) T.-S. Wong, et al., Eur. J. Cancer 2003, 39, 1881-1887. (d) Y. Delpu, et al. Int J Mol Sci. 2013, 14, 15029-15058.

4. N. Plongthongkum, D. H. Diep, K. Zhang, Nat. Rev. Genet. 2014, 15, 647-661.

5. (a) Y. Wang, E. J. H. Wee, M. Trau, Chem. Commun. 2016, 52, 3560-3563. (b) E. J. H. Wee, T. H. Ngo, M. Trau, Sci. Rep. 2015, 5, article number 15028.

6. (a) K. M. Koo, E. J. H. Wee, et al. Biosens. Bioelectron. 2014, 56, 278-285. (b) E. J. H. Wee, S. Rauf, et al. Clin. Chem. 2015, 61, 163-171. (c) L. G. Carrascossa, A. A. I. Sina, et al. Chem. Commun. 2014, 50, 3585-3588.

7. (a) D. Kato, K. Goto, et al. Anal. Chem. 2011, 83, 7595-7599. (b) D. Kato, N. Sekioka, et al. J. Am. Chem. Soc. 2008, 130, 3716-3717. (c) R. Kurita, K. Arai, et al. Anal. Chem. 2012, 84, 1799-1803.

8. (a) A. A. I. Sina, et al. Chem. Commun. 2014, 50, 1315313156.(b) K. M. Koo, et al. Analyst 2014, 139, 6178-6184

9. N. Varghese, U. Mogera, A. Govindaraj, A. Das, P. Maiti, A. K. Sood, C. N. R. Rao, ChemPhysChem 2009, 10, 206-210.

10. M. Wu, R. Kempaiah, et al. Langmuir 2011, 27, 2731-2738.

11. S. Gowtham, R. H. Scheicher, et al. Phys. Rev. B 2007, 76, article number 033401.

12. J. Zhang, L. Wang, D. Pan, S. Song, C. Fan, Chem. Commun. 2007, 1154-1156.

13. J. Das, et al. Nat. Chem. 2012, 4, 642-648.

14. (a) W. K. Tang, C. H. Chui, et al., Int. J. Mol. Med. 2007, 19, 915-923. (b) K. Kase, V. Gopalan, et al. Exp Cell Res. 2014, 326, 166-173.

15. (a) C. Long, et al., Cancer Invest. 2007, 25, 685-690. (b) T. Kuroki, et al. Clin. Cancer Res. 2003, 9, 1441-1445. 